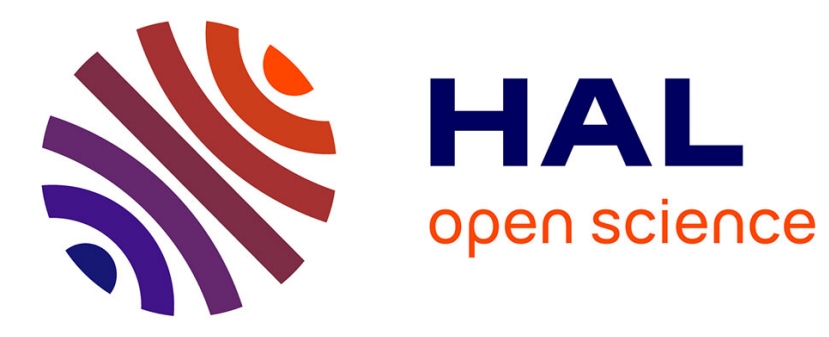

\title{
Bilan d'érosion d'un cours d'eau alpin : l'Isère à Grenoble (France)
}

\author{
Dominique Dumas
}

\section{To cite this version:}

Dominique Dumas. Bilan d'érosion d'un cours d'eau alpin: l'Isère à Grenoble (France). Annales de Géomorphologie / Annals of Geomorphology / Zeitschrift für Geomorphologie, 2008, 52 (1), pp.85-103. halshs-00281933

\section{HAL Id: halshs-00281933 \\ https://shs.hal.science/halshs-00281933}

Submitted on 1 Mar 2009

HAL is a multi-disciplinary open access archive for the deposit and dissemination of scientific research documents, whether they are published or not. The documents may come from teaching and research institutions in France or abroad, or from public or private research centers.
L'archive ouverte pluridisciplinaire HAL, est destinée au dépôt et à la diffusion de documents scientifiques de niveau recherche, publiés ou non, émanant des établissements d'enseignement et de recherche français ou étrangers, des laboratoires publics ou privés. 
D. Dumas, 2008. Bilan d'érosion d'un cours d'eau alpin : l'Isère à Grenoble (France). Zeitschrift für Geomorphologie, vol. 52, number 1, 85-103.

\title{
Bilan d'érosion d'un cours d'eau alpin : I'lsère à Grenoble (France) Erosion data of a major alpine river: the Isère River at Grenoble (France)
}

Dominique Dumas, Institut de Géographie Alpine, Univ. Joseph Fourier, 14 bis, av. Marie-Reynoard, 38100 Grenoble, France dominique.dumas@uif-grenoble.fr

\section{Résumé}

Le bassin de l'Isère, en amont de Grenoble, apporte au Rhône une contribution importante de matières en suspension. Par ailleurs, la surface de ce bassin versant en fait un témoin révélateur de la dynamique érosive actuelle dans les Alpes du Nord. Un équipement pour l'étude des flux de matière dissoute totale (MDT) et de matière en suspension (MES), a été installé progressivement à Grenoble, depuis 1994. Les résultats présentés sont établis à partir de mesures systématiques. En effet, dans les Alpes, il n'existe encore dans ce domaine que peu de données continues, et relevées sur une décennie. Ces mesures permettent de dresser un bilan d'érosion sur le bassin de l'Isère, et d'estimer l'érosion totale à $3.73 \mathrm{Mt} \mathrm{an}^{-1}$ et un taux d'érosion spécifique moyen de 652 $\mathrm{t} \mathrm{km}^{-2} \mathrm{an}^{-1}$. L'érosion fluviatile moyenne avec $2.0 \mathrm{Mt} \mathrm{an}^{-1}$ est légèrement plus forte que celle issue des processus chimiques dont la valeur moyenne est estimée à $1.73 \mathrm{Mt} \mathrm{an}^{-1}$. Mais la variabilité inter-annuelle de ces flux reste forte, et tout particulièrement le flux de matière en suspension. Le transit annuel de MES est très supérieur à celui de MDT lors des années connaissant des crues importantes. L'exportation de matière dissoute reste cependant considérable et traduit bien l'importance des processus de dissolution au sein du bassin.

\begin{abstract}
The Isère River is one of the major suppliers of suspended matter to the Rhône River. In addition, the large area covered by the Isère River Basin makes it a particularly valuable source of information about current sedimentary dynamics in the Northern Alps. Instruments for studying the flow of total dissolved matter (TDM) and suspended matter (SM) have been set up step by step since 1994. The results presented here are the first to be based on the systematic measurement of a major Alpine watercourse. Decade-long data of this type are scarce for the Alpine area. The present measurements have enabled a sedimentary assessment of the Isère Basin to be made; total erosion was estimated at $3.73 \mathrm{Mt} \mathrm{yr}^{-1}$ and the mean specific erosion rate at $652 \mathrm{t} \mathrm{km}^{-2} \mathrm{yr}^{-1}$. Mean fluvial erosion at $2.0 \mathrm{Mt} \mathrm{yr}^{-1}$ is slightly greater than that caused by chemical processes, whose mean value is estimated at 1.73 $\mathrm{Mt}_{\mathrm{yr}}{ }^{-1}$. Rates of erosion vary widely from year to year, however, especially rergarding suspended matter. The annual amount of SM thus greatly exceeds TDM in years when the flow is very high. The amount of dissolved matter exported nevertheless remains considerable and is a reliable indicator of the importance of dissolution processes within the basin.
\end{abstract}

\section{INTRODUCTION}

Le transit sédimentaire fluviatile a des impacts économiques. En effet, il impose un entretien régulier des cours d'eau. II favorise, entre autres, l'exhaussement des lits, affectant ainsi directement le niveau de protection des digues, ou encore l'envasement des retenues, limitant une " gestion durable " de I'hydroélectricité. Aussi, en terme de gestion, la quantification des flux actuels est essentielle. Or I'Isère, par le caractère montagneux de son bassin, est l'un des principaux pourvoyeurs de matière en suspension du Rhône. L'objectif de cette étude est de quantifier aux pas de temps mensuel et annuel la quantité de sédiments exportés par l'Isère à Grenoble. L'indigence de la charge de fond exportée à Grenoble (Salvador, 1991 ; Vautier, 2000), permet de cerner l'ensemble des transports à partir de mesures bi-quotidiennes des matières en suspension (MES) et des matières dissoutes (MDT). La dynamique sédimentaire moyenne globale d'un bassin montagneux peut ainsi être dégagée. Même si les taux d'érosion établis à partir de ces exportations restent indicatifs, et ne peuvent totalement traduire l'efficacité de l'érosion actuelle réelle. Car, d'une part, compte-tenu de son contexte montagneux et de sa superficie, de près de $6000 \mathrm{~km}^{2}$, le bassin de l'Isère présente une forte hétérogénéité spatiale. II intègre aussi bien des zones faiblement érodées que des zones où la dynamique érosive est intense. D'autre part, ces vitesses d'érosion moyennes ne peuvent que difficilement faire la part du stockage et de la remobilisation des sédiments dans le lit du cours d'eau et des ses affluents. Des travaux sur les profils de l'Isère dans la vallée du Grésivaudan (Vautier, 2000 ; Allain Jegou, 2002) montrent cependant qu'il n'y a aucune commune mesure entre les flux associés au stockage et au déstockage et ceux liés à l'exportation dont les valeurs sont incomparablement plus élevées. 


\section{CONTEXTE GEOGRAPHIQUE DU BASSIN DE L'ISERE}

Situé dans les Alpes du Nord, le bassin de l'Isère, en amont de Grenoble (figure 1), couvre une superficie de $5720 \mathrm{~km}^{2}$ en intégrant les massifs de la Vanoise, du Beaufortin, de Belledonne et le rebord oriental des Préalpes du Nord (Chartreuse, Bauges, Aravis). Depuis plus de 150 ans, des observations limnimétriques au quai Perrière de Grenoble, puis une station de mesure, installée en 1960, permettent de retracer le comportement hydrologique de l'Isère. Le module hydrologique de l'Isère est de $183 \mathrm{~m}^{3} \mathrm{~s}^{-1}$, pour une période de 46 années, depuis 1960, date de création de la station hydrologique utilisée dans cette étude (Diren, Banque Hydro : W1410010). Au cours du siècle dernier, l'Isère est restée relativement calme, puisque les plus puissantes crues observées ont à peine dépassé la moitié du débit maximal estimé, de $2000 \mathrm{~m}^{3} \mathrm{~s}^{-1}$, lors de la grande crue de 1859 (Dumas, 2004b). On estime généralement (Vautier, 2000, Banque Hydro - Diren) que la crue décennale se situe à $760 \mathrm{~m}^{3} \mathrm{~s}^{-1}$, à près de $1000 \mathrm{~m}^{3} \mathrm{~s}^{-1}$ pour la crue cinquantennale, et à plus de $1600 \mathrm{~m}^{3} \mathrm{~s}^{-1}$ pour la crue centennale (ces valeurs seraient néanmoins un peu plus élevées selon d'autres études (Vivian, 1969 ; Allain Jegou, 2002). Le régime nivo-pluvial, avec de hautes eaux de mai à juillet, traduit le caractère montagneux de son bassin dont plus de $70 \%$ sont situés au-dessus de $1000 \mathrm{~m}$ et $17 \%$ audessus de 2500 m d'altitude.

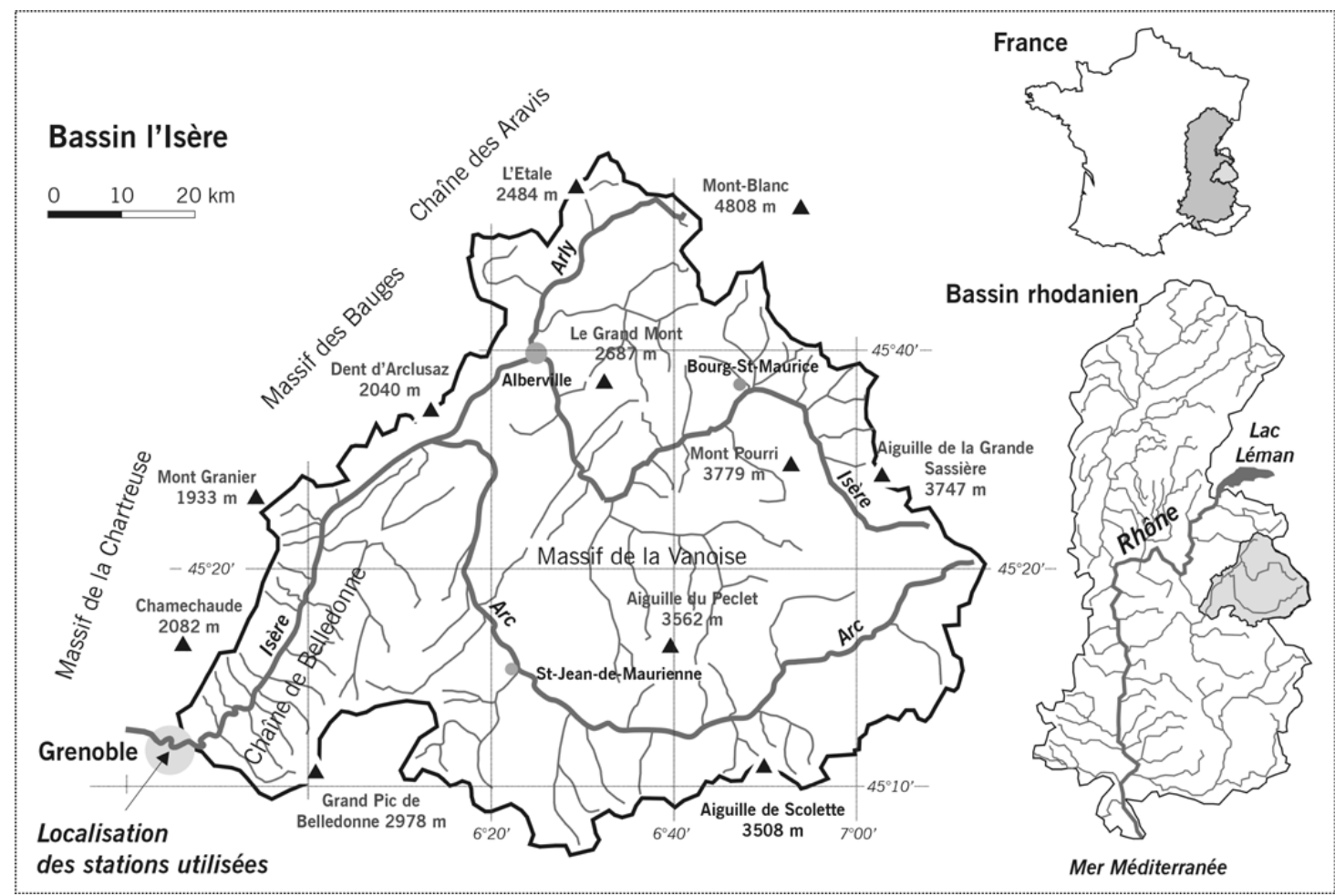

Figure 1. Bassin versant de l'Isère à Grenoble.

Figure 1. The Isère River Basin upstream from Grenoble.

\section{MÉTHODOLOGIE}

Sur le Campus de Grenoble, un équipement pour l'étude des flux de matière dissoute totale (MDT) et de matière en suspension (MES), a été installé progressivement depuis 1994 (Peiry, 1997). Un préleveur automatique permet d'analyser des échantillons d'eau sur lesquels sont mesurées les concentrations en MES et MDT, obtenues respectivement par filtration-pesée (membranes de 0.45 $\mu \mathrm{m})$ et indirectement avec une mesure de la conductivité de l'eau. La concentration des matières dissoutes est ensuite appréciée par le biais de coefficients dont la valeur est liée aux gammes de mesure de la conductivité (Rodier, 1996).

Les prélèvements sont effectués dans la zone de traitement des eaux usées de l'agglomération grenobloise, en amont de la station d'épuration et à une dizaine de kilomètres en aval de la limite de cette zone, ce qui réduit une éventuelle influence des apports anthropiques sur les mesures de la matière dissoute. Par ailleurs, les flux spécifiques journaliers de MDT, calculés à partir de 
prélèvements non continus depuis 1971 (Agence de l'Eau Rhône-Méditerranée et Corse), présentent des valeurs et des variations sensiblement identiques à Pontcharra (station 141000), située à $40 \mathrm{~km}$ à l'amont de Grenoble, à Grenoble et à Tullins, située $30 \mathrm{~km}$ à l'aval de Grenoble (station 147130). Cette similitude des valeurs dans l'espace et dans le temps tend à montrer une influence réduite des apports anthropiques, qui seraient sinon vraisemblablement localisés et irréguliers. Les flux spécifiques de MDT sont analogues à ceux observés à Grenoble, avec une moyenne annuelle inscrite entre $0.70 \mathrm{t} \mathrm{km}^{-2} \mathrm{j}^{-1}$ et $0.75 \mathrm{t} \mathrm{km}^{-2} \mathrm{j}^{-1}$.

Les mesures des MES ont débuté en janvier 1995, et celles de la MDT en janvier 2000. Elles étaient bi-quotidiennes de 1995 à 2004 et sont quotidiennes à partir de janvier 2005. Parallèlement à ces mesures systématiques, plus d'une quinzaine de jaugeages complets ont permis de confronter les mesures ponctuelles aux concentrations moyennes réelles sur la section (Dumas, 2004a). Ces mesures ont été conduites pour un débit de l'Isère inscrit entre $105 \mathrm{~m}^{3} \mathrm{~s}^{-1}$ (le 10 octobre 2002) et 348 $\mathrm{m}^{3} \mathrm{~s}^{-1}$ (le 17 mai 2001). Ainsi, la méthode retenue pour l'estimation de la concentration moyenne sur l'ensemble de la section $(\mathrm{Cm})$, à partir de la concentration de l'échantillon prélevé, utilise le modèle linéaire suivant (équation 1 ) :

$\mathrm{Cm}=0.832 \mathrm{X}+18.46 \quad \mathrm{R}^{2}=0.94$ (équation 1 )

où $X=$ mesure ponctuelle de la MES (dispositif de prélèvement)

Une moyenne journalière de la concentration ainsi étendue à toute la section mouillée est ensuite évaluée à partir des deux prélèvements quotidiens. Cette correction, induite par les gradients de turbidité des flux de MES, n'est pas nécessaire pour les MDT, qui présentent toujours une répartition relativement homogène des valeurs sur l'ensemble de la section.

\section{RÉSULTATS Les flux de matière dissoute}

De 2000 à 2005, la concentration journalière totale de l'Isère en minéraux dissous est comprise entre 186 et $509 \mathrm{mg} \mathrm{L}^{-1}$. L'évaluation de ces concentrations totales traduit essentiellement l'extension, dans le bassin, des roches carbonatées (schistes lustrés, calcaires, dolomies), et des roches sulfatées (gypses). Ainsi, les analyses géochimiques des matières dissoutes à Grenoble, conduites par le Service Eau de la D.D.E., montrent la part majoritaire des carbonates, avec plus de $54 \%$ de la composition chimique des eaux, puis des sulfates, avec 36 à $37 \%$ des éléments dissous (tableau 1). Ces compositions chimiques ne sont cependant qu'indicatives, car elles sont issues de prélèvements non continus, et mal répartis sur une année moyenne, puisque, par exemple, sur les 43 échantillons retenus, 18 se placent en février-mars, et 17 en septembre. Or, les concentrations totales, de 330 $\mathrm{mg} \mathrm{L}^{-1}$ en moyenne sur une année (tableau 2), présentent une fluctuation saisonnière qui imposerait, en toute rigueur, pour obtenir des valeurs caractéristiques, un suivi relativement régulier de la composition chimique des eaux. C'est pendant la période des basses eaux que les concentrations mensuelles moyennes sont maximales (en décembre, $378 \mathrm{mg} \mathrm{L}^{-1}$ ), du fait d'une dilution moins importante des matières dissoutes. A l'inverse, la période des hautes eaux marque une baisse des concentrations (en mai, $268 \mathrm{mg} \mathrm{L}^{-1}$ )

Tableau 1. Débit instantané $\left(m^{3} \mathrm{~s}^{-1}\right)$ et concentration des sels dissous $\left(\mathrm{mg} \mathrm{L}^{-1}\right)$ de l'lsère à Grenoble centre-ville (station $n^{\circ} 142000$ ) et à Grenoble campus (station $n^{\circ} 141900$ ). Données : Agence de l'Eau Rhône-Méditerranée et Corse.

Table 1. Isère River flow in $\mathrm{m}^{3} \mathrm{~s}^{-1}$ and concentration of dissolved mineral salts ( $\mathrm{mg} \mathrm{L}^{-1}$ ) at Grenoble city centre (Station $n^{\circ 142000)}$ and Grenoble campus (Station $\left.n^{\circ} 141900\right)$. Data source: Agence de l'Eau Rhône-Méditerranée et Corse.

\begin{tabular}{|c|c|c|c|c|c|c|c|c|c|c|c|}
\hline & & Débit & SO4-- & $\mathrm{HCO}-$ & $\mathrm{Ca}++$ & $\mathrm{Mg}++$ & $\mathrm{Cl}-$ & $\mathrm{Na}+$ & NO3- & $\mathrm{K}_{+}$ & $\mathrm{PO4}$ \\
\hline Grenoble-ville & Valeur min & 75 & 105 & 119 & 74 & 5,8 & 7,0 & 4,8 & 1,0 & 0,8 & 0,01 \\
\hline (station 142.000): & Valeur max & 245 & 200 & 180 & 100 & 21,0 & 17,0 & 14,0 & 3,4 & 2,0 & 0,90 \\
\hline 13 observations entre & Moyenne & 130 & 153,6 & 136,8 & 88,8 & 12,4 & 11,5 & 8,9 & 2,1 & 1,2 & 0,26 \\
\hline 1971 et 1981 & $\%$ & & $37,0 \%$ & $32,9 \%$ & $21,4 \%$ & $3,0 \%$ & $2,8 \%$ & $2,1 \%$ & $0,5 \%$ & $0,3 \%$ & $0,06 \%$ \\
\hline Grenoble-campus & Valeur min & 80 & 68 & 98 & 50 & 3,1 & 5,3 & 3,9 & 1,1 & 0,8 & 0,03 \\
\hline (station 141.900) : $\quad 30$ & Valeur max & 293 & 223 & 195 & 116 & 42,2 & 17,0 & 11,8 & 3,9 & 1,7 & 0,47 \\
\hline observations entre & Moyenne & 147 & 150,4 & 141,2 & 90,0 & 13,0 & 10,9 & 7,0 & 2,4 & 1,2 & 0,15 \\
\hline 1987 et 2002 & $\%$ & & $36,1 \%$ & $33,9 \%$ & $21,6 \%$ & $3,1 \%$ & $2,6 \%$ & $1,7 \%$ & $0,6 \%$ & $0,3 \%$ & $0,04 \%$ \\
\hline
\end{tabular}


Si l'exportation des matières dissoutes reste largement tributaire de l'écoulement (Martin, 1987), la relation inverse des concentrations moyennes avec les débits atténue les variations saisonnières des flux de MDT. Ainsi, sur la période 2000-2005, la variation saisonnière des écoulements est de 2.2, contre une variation de 1.7 pour les flux mensuels moyens de MDT (tableau 2).

Sur la période 2000-2005, les mesures permettent de définir le régime du transport de matière dissoute par les quantiles des distributions statistiques des MDT pour chaque mois de l'année (tableau 2). Les concentrations et les flux de MDT montrent, quel que soit le quantile observé, une grande stabilité de ces paramètres et la relative indigence des variations saisonnières, toujours inférieures à 2 ordres de grandeur.

Tableau 2. Régime des concentrations des matières dissoutes (données 2000-2005)

Table 2. Concentrations of dissolved matter (2000-2005)

\begin{tabular}{|c|c|c|c|c|c|c|c|c|c|c|c|}
\hline & \multirow{2}{*}{$\begin{array}{l}\text { Q moy } \\
(\mathrm{m} 3 / \mathrm{s})\end{array}$} & \multicolumn{4}{|c|}{ Concentration journalière en MD (mg/L) } & \multirow{2}{*}{$\begin{array}{c}\text { Flux moyen } \\
\text { (tonnes) }\end{array}$} & \multicolumn{5}{|c|}{ Flux spécifique $\left(T / \mathrm{km}^{2} / \mathrm{j}\right)$} \\
\hline & & moyenne & $\mathrm{C} 10$ & $\mathrm{C} 50$ & $\mathrm{C} 90$ & & moy & $\mathrm{C} 10$ & $\mathrm{C} 50$ & $\mathrm{C} 90$ & $\max$ \\
\hline $\mathrm{J}$ & 142 & 358 & 295 & 369 & 406 & 130489 & 0,736 & 0,402 & 0,746 & 0,999 & 2,420 \\
\hline $\mathrm{F}$ & 134 & 356 & 280 & 364 & 433 & 114529 & 0,707 & 0,478 & 0,717 & 0,907 & 1,130 \\
\hline M & 175 & 333 & 268 & 329 & 395 & 151143 & 0,848 & 0,564 & 0,793 & 1,141 & 2,546 \\
\hline A & 172 & 334 & 271 & 334 & 392 & 144493 & 0,842 & 0,614 & 0,815 & 1,153 & 1,471 \\
\hline M & 255 & 268 & 227 & 267 & 309 & 178229 & 1,005 & 0,758 & 0,972 & 1,322 & 1,751 \\
\hline J & 250 & 261 & 220 & 260 & 296 & 164530 & 0,959 & 0,724 & 0,917 & 1,259 & 1,649 \\
\hline $\mathrm{J}$ & 165 & 306 & 224 & 309 & 363 & 128908 & 0,727 & 0,533 & 0,712 & 0,993 & 1,235 \\
\hline A & 141 & 320 & 271 & 318 & 377 & 116922 & 0,659 & 0,471 & 0,634 & 0,846 & 1,584 \\
\hline $\mathrm{s}$ & 119 & 341 & 290 & 339 & 396 & 102446 & 0,597 & 0,420 & 0,592 & 0,776 & 1,041 \\
\hline O & 132 & 342 & 284 & 346 & 398 & 116881 & 0,659 & 0,388 & 0,610 & 0,969 & 1,973 \\
\hline $\mathrm{N}$ & 131 & 358 & 303 & 365 & 399 & 116440 & 0,679 & 0,410 & 0,609 & 1,033 & 1,548 \\
\hline $\mathrm{D}$ & 112 & 378 & 342 & 373 & 416 & 113518 & 0,640 & 0,373 & 0,637 & 0,960 & 1,340 \\
\hline A & 161 & 330 & 256 & 333 & 396 & 1577788 & 0,755 & 0,467 & 0,731 & 1,066 & 2,546 \\
\hline
\end{tabular}

En utilisant une relation de type exponentiel, ajustée aux concentrations journalières moyenne de MDT (MDTj) et aux débits journaliers (Qj), il est possible de reconstituer d'une manière assez sûre, les flux de MDT sur la période pour laquelle des mesures de MES ont été effectuées, puis sur une période plus longue. La relation établie est de la forme (équation 2, figure 2) :

$\mathrm{MDTj}=1411.6 \mathrm{Qj}^{-0.2947} \mathrm{R}=0.75$ (équation 2)

Cette relation souligne le régime en dilution des concentrations de l'Isère.

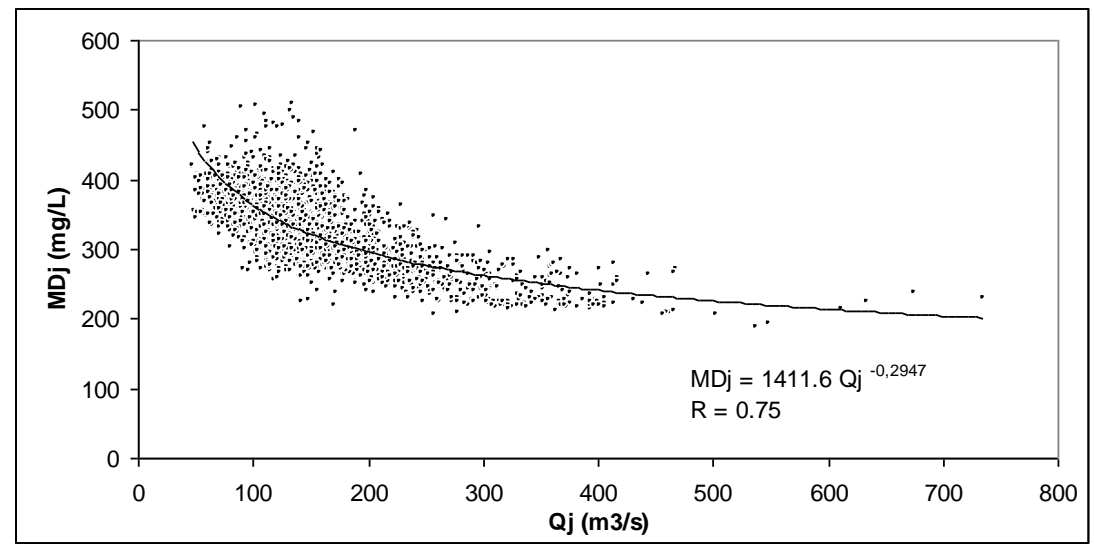

Figure 2. Relation des concentrations journalières de MDT avec les débits journaliers

Figure 2. Comparison of daily TDM concentrations and daily flow rates

En appliquant l'équation 2, il est possible de reconstituer les concentrations journalières depuis 1995. Sur la période mesurée, la charge totale annuelle moyenne dissoute est alors de $327 \mathrm{mg} \mathrm{L}^{-1}$ avec les valeurs reconstituées, contre $330 \mathrm{mg} \mathrm{L}^{-1}$ obtenus à partir des mesures quotidiennes (tableau 3). A l'échelle annuelle, les concentrations et les flux de MDT calculés présentent des écarts avec les valeurs mesurées qui ne dépassent pas respectivement $13 \mathrm{mg} \mathrm{L}^{-1}$ et $51000 \mathrm{t} \mathrm{an}^{-1}$. Sur la période 1995-2005, l'évaluation du transfert annuel moyen des substances dissoutes est de $1.6 \mathrm{Mt} \mathrm{an}^{-1}$, pour 
un débit annuel moyen de $170 \mathrm{~m}^{3} \mathrm{~s}^{-1}$ (tableau 3, figure 3). Le transit moyen de matériaux dissous est cependant légèrement supérieur, avec $1.7 \mathrm{Mt} \mathrm{an}^{-1}$, sur la période 1960 à 2005 qui est actuellement utilisée pour définir le module interannuel moyen caractéristique de l'Isère à Grenoble $\left(183 \mathrm{~m}^{3} \mathrm{~s}^{-1}\right)$.

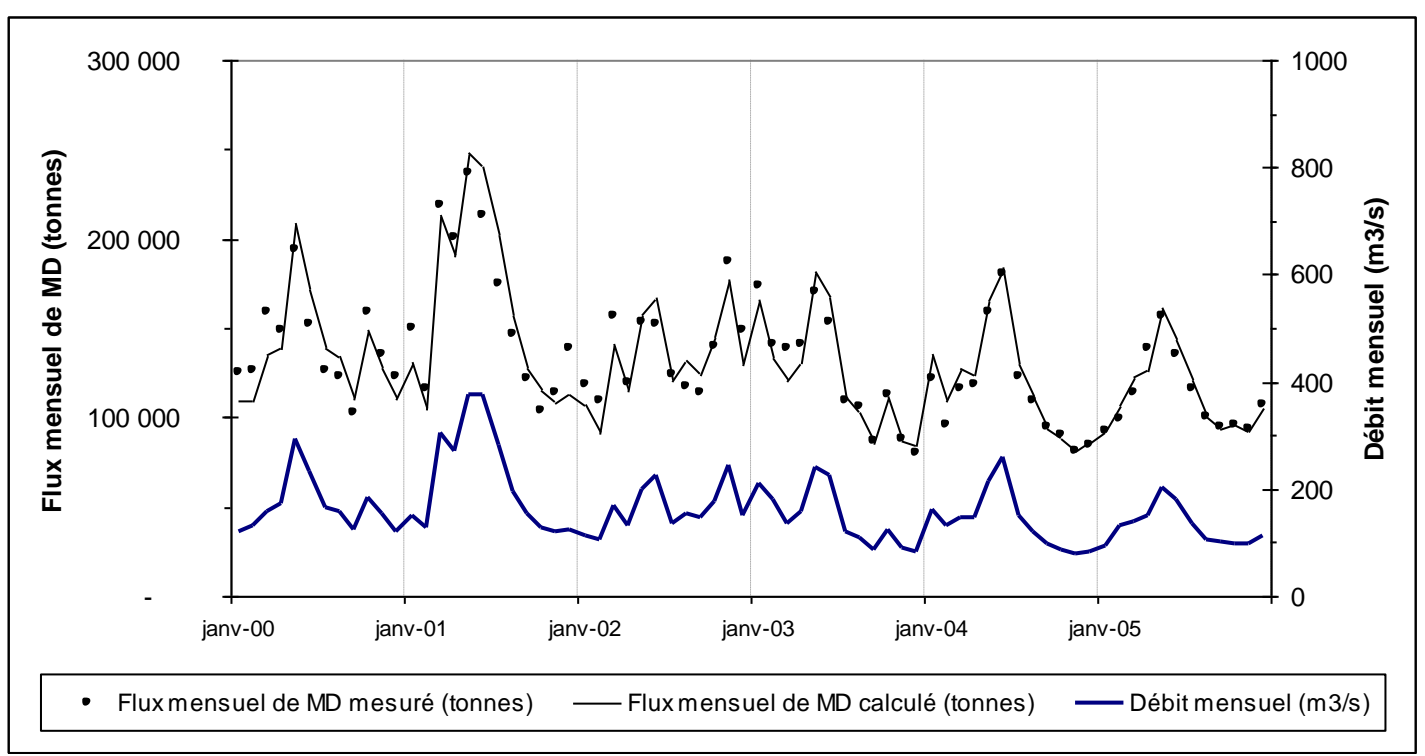

Figure 3. Variation mensuelle des flux de MDT et des débits, de 2000 à 2005. (valeurs débits : Banque Hydro)

Figure 3. Monthly variations in TDM flows and river flow rates, 2000 - 2005. (flow rate data source: Banque Hydro)

Tableau 3. Concentration et flux de MDT annuels mesurés et calculés à partir du modèle journalier

Table 3. Concentration and flow of annual TDM measured and calculated from the daily model

\begin{tabular}{|c|c|c|c|c|c|}
\hline Année / Période & $\begin{array}{l}\text { Module } \\
(\mathrm{m} 3 / \mathrm{s})\end{array}$ & \multicolumn{2}{|c|}{$\begin{array}{l}\text { Concentration de } \mathrm{MD}(\mathrm{mg} / \mathrm{L}) \\
\text { mesurée calculée }\end{array}$} & \multicolumn{2}{|c|}{ Flux de MD (tonnes) } \\
\hline 1995 & 235 & & 296 & & 2040773 \\
\hline 1996 & 145 & & 332 & & 1480019 \\
\hline 1997 & 148 & & 334 & & 1485392 \\
\hline 1998 & 158 & & 325 & & 1563722 \\
\hline 1999 & 213 & & 302 & & 1910748 \\
\hline 2000 & 168 & 330 & 319 & 1672719 & 1634355 \\
\hline 2001 & 218 & 306 & 303 & 1934682 & 1936088 \\
\hline 2002 & 163 & 331 & 321 & 1641234 & 1599328 \\
\hline 2003 & 147 & 341 & 335 & 1501873 & 1476013 \\
\hline 2004 & 140 & 329 & 342 & 1372641 & 1423399 \\
\hline 2005 & 129 & 340 & 344 & 1343577 & 1354303 \\
\hline Moy. 2000-2005 & 161 & 330 & 327 & 1577788 & 1570581 \\
\hline Moy. 1995-2005 & 170 & - & 323 & - & 1627649 \\
\hline
\end{tabular}

Au-delà, des coefficients de corrélation élevés, ces différentes estimations apparaissent relativement robustes puisqu'aux échelles mensuelle et annuelle, le transit de MDT reste toujours fortement tributaire des écoulements. D'ailleurs, le solidigramme mensuel est relativement semblable à l'hydrogramme (figure 3). En outre, à l'échelle annuelle, ces estimations sont facilitées par la faiblesse de la variabilité interannuelle de ces flux dissous, d'un rapport inférieur à 2 . L'effet amplificateur des crues sur l'exportation de matériaux, jouant pleinement sur le transport solide de MES, n'est guère opérant sur le transport dissous (figure 4). 


\section{Matières en suspension}

\section{Analyse des flux sédimentaires des MES}

Les mesures quotidiennes de la MES se sont échelonnées de 1995 à 2005, mais les années 1995 et 2005 doivent être écartées des statistiques mensuelles et annuelles, car l'exportation des matières particulaires fut alors trop fortement tributaire d'une mobilisation consécutive à divers aménagements proches du cours d'eau.

Ainsi, les travaux engagés dès 1994 pour la construction de l'autoroute de la Maurienne, dans la vallée de l'Arc, avec le confortement des digues et la construction d'ouvrages d'art (notamment les viaducs d'Aiton, de Saint André et d'Escalade), ont fortement contribué à perturber les flux sédimentaires quotidiens observés en 1995. Par exemple, c'est entre le $1^{\text {er }}$ et le 4 juin 1995 , à l'occasion d'une crue ayant remobilisé un stock sédimentaire généré par ces travaux, que les flux quotidiens de MES maximaux ont été mesurés, avec 370000 à 430000 tonnes de sédiments évacués par jour (flux spécifique de 65 à $76 \mathrm{t} \mathrm{km}^{-2} \mathrm{j}^{-1}$ ). De même, en 2005, des travaux d'aménagement des digues, à $1 \mathrm{~km}$ environ en amont des prélèvements d'eau (commune de Gières), de janvier 2005 à juillet 2006, ont également fortement perturbé les mesures. On relève cette année là, le 18 avril 2005, la concentration moyenne journalière la plus élevée $(\mathrm{Cmj})$, observée depuis la mise en place des capteurs, avec plus de $15.5 \mathrm{~g} \mathrm{~L}^{-1}$ en moyenne sur la section (flux spécifique de 28.9 $\left.\mathrm{t} \mathrm{km}^{-2} \mathrm{j}^{-1}\right)$.

Sur la période 1996 à 2004, la moyenne, les déciles et la médiane des concentrations, ainsi que les flux moyens, calculés à partir des valeurs quotidiennes, permettent de caractériser le régime du transport sédimentaire de l'Isère (Meybeck, 2001). La variabilité saisonnière des concentrations et des flux sédimentaires se cale assez bien dans l'ensemble sur le régime hydrologique de l'Isère (tableau 4). La variation mensuelle maximale des concentrations moyennes est de 4.2 , mais elle est plus élevée sur les déciles supérieurs inscrits entre 143 et $849 \mathrm{mg} \mathrm{L}^{-1}$ (variation mensuelle maximale de 5.9).

Les flux spécifiques moyens, avec un pic principal en mai et un pic secondaire en mars, caractérisent moins bien ce régime, car ils sont trop tributaires de transferts sédimentaires liés à des crues. Les médianes et les déciles supérieurs sont plus pertinents. Ils montrent une forte variabilité saisonnière des transits sédimentaires, avec un rapport maximal de 6.5 pour les médianes, et de 15.4 pour les déciles supérieurs (tableau 4:0.3 à $5.1 \mathrm{t} \mathrm{km}^{2} \mathrm{j}^{-1}$, soit un transit mensuel variant de 52372 à 899544 $\mathrm{t}$ mois $\left.{ }^{-1}\right)$. Les déciles supérieurs très élevés en mai et en juin montrent clairement l'importance de la fusion nivale dans la production sédimentaire.

Tableau 4. Régime saisonnier des débits et du transport de MES pour la période 1996 à 2004 C10, C90, C50 : déciles et médianes des concentrations journalières et des flux spécifiques journaliers de MES. Comme le flux moyen, ils sont calculés à partir des données moyennes journalières.

Table 4. Seasonal river flow and SM transport, 1996 - 2004

C10, C90, C50: deciles and medians of daily concentrations and specific daily SM flows. Like mean flow, they were calculated from mean daily data.

\begin{tabular}{|c|c|c|c|c|c|c|c|c|c|c|c|}
\hline & \multirow{2}{*}{$\begin{array}{l}\text { Q moy } \\
(\mathrm{m} 3 / \mathrm{s})\end{array}$} & \multicolumn{4}{|c|}{ Concentration journalière MES (mg/L) } & \multirow{2}{*}{$\begin{array}{l}\text { Flux moyen } \\
\text { (tonnes) }\end{array}$} & \multicolumn{5}{|c|}{ Flux spécifique $\left(T / \mathrm{km}^{2} / \mathrm{j}\right)$} \\
\hline & & moy & $\mathrm{C} 10$ & C50 & $\mathrm{C} 90$ & & moy & $\mathrm{C} 10$ & C50 & $\mathrm{C} 90$ & $\max$ \\
\hline $\mathrm{J}$ & 141,2 & 125,0 & 29,8 & 62,4 & 162,5 & 91481 & 0,516 & 0,05 & 0,13 & 0,44 & 62,97 \\
\hline $\mathrm{F}$ & 134,2 & 90,4 & 34,3 & 61,4 & 143,3 & 36705 & 0,229 & 0,05 & 0,12 & 0,33 & 7,50 \\
\hline $\mathrm{M}$ & 161,5 & 244,6 & 37,9 & 79,6 & 337,6 & 208566 & 1,176 & 0,06 & 0,18 & 1,06 & 52,46 \\
\hline A & 169,9 & 178,4 & 49,2 & 107,8 & 362,9 & 97780 & 0,570 & 0,09 & 0,25 & 1,28 & 7,18 \\
\hline M & 265,4 & 367,1 & 69,2 & 185,2 & 848,6 & 366330 & 2,066 & 0,19 & 0,62 & 5,07 & 39,57 \\
\hline $\mathrm{J}$ & 257,1 & 383,1 & 95,1 & 203,3 & 804,9 & 294333 & 1,715 & 0,28 & 0,76 & 4,19 & 22,41 \\
\hline $\mathrm{J}$ & 180,9 & 199,5 & 71,0 & 124,0 & 323,1 & 113220 & 0,639 & 0,14 & 0,32 & 1,07 & 26,89 \\
\hline A & 141,0 & 235,9 & 71,8 & 144,5 & 433,7 & 95356 & 0,538 & 0,13 & 0,30 & 1,14 & 7,99 \\
\hline $\mathrm{S}$ & 125,5 & 165,7 & 61,4 & 123,5 & 256,1 & 56342 & 0,328 & 0,10 & 0,24 & 0,63 & 6,13 \\
\hline $\mathrm{O}$ & 140,6 & 148,6 & 56,9 & 109,2 & 270,2 & 60515 & 0,341 & 0,09 & 0,20 & 0,64 & 3,39 \\
\hline $\mathrm{N}$ & 147,9 & 151,8 & 46,2 & 86,5 & 254,8 & 66057 & 0,385 & 0,07 & 0,17 & 0,71 & 2,52 \\
\hline $\mathrm{D}$ & 134,2 & 125,4 & 43,9 & 92,8 & 195,6 & 56753 & 0,320 & 0,07 & 0,15 & 0,44 & 19,62 \\
\hline A & 166,6 & 201,3 & 46,2 & 108,7 & 367,3 & 1543438 & 0,739 & 0,08 & 0,24 & 1,25 & 62,97 \\
\hline
\end{tabular}




\section{Importance des crues dans l'exportation des MES}

Sur l'Isère, comme sur la plupart des cours d'eau (Walling et al., 1992 ; Batalla et al., 1995 ; Serrat, 1999 ; Serrat et al., 2001, Pont et al., 2002 ; Meybeck et al., 2003), les grandes crues sont les évènements qui marquent l'essentiel du transit sédimentaire annuel. Trois crues d'une période de retour égale ou légèrement supérieure à 10 ans, ont été observées à Grenoble ; en mai 1999 (Qmax = $\left.809 \mathrm{~m}^{3} \mathrm{~s}^{-1}\right)$, en mars $2001\left(\mathrm{Qmax}=875 \mathrm{~m}^{3} \mathrm{~s}^{-1}\right)$ et en janvier $2004\left(\mathrm{Qmax}=750 \mathrm{~m}^{3} \mathrm{~s}^{-1}\right)$, avec un transit de plus de 1 million de tonnes, en dix jours, pour les deux premières, et d'un demi-million de tonnes, en quatre jours, pour la troisième. Sur ces brèves durées, elles ont évacué respectivement $34 \%$, $39 \%$ et $30 \%$ du tonnage annuel de sédiments pour seulement $1.3 \%, 5.4 \%$ et $3.3 \%$ des volumes annuels écoulés.

Les courbes de durée annuelle, « duration curves » (Meybek et al., 2003), permettent de mieux cerner l'importance de ces évènements et le caractère particulier du transit de MES par rapport à l'écoulement ou à l'exportation de matière dissoute (figure 4). Sur cette figure, afin de conserver une bonne lisibilité, seules les courbes extrêmes ont été positionnées, les autres restent relativement similaires, "empilées » les unes sur les autres, et inscrites dans le biseau limité par les années 2000 et 2004 pour les flux de MES. Les années 1995 et 2005 présentent en revanche des courbes de durée atypiques, et n'ont pas été représentées sur la figure 4. En 30 jours, par exemple, soit une durée d'environ $8 \%$ d'une année, plus de la moitié du transit de MES annuel est généralement évacuée par l'Isère (entre $44 \%$ et $71 \%$ selon les années, et $55 \%$ en moyenne).

L'importance des flux lors de ces évènements favorise une grande variabilité inter-annuelle des exportations annuelles de matière en suspension.

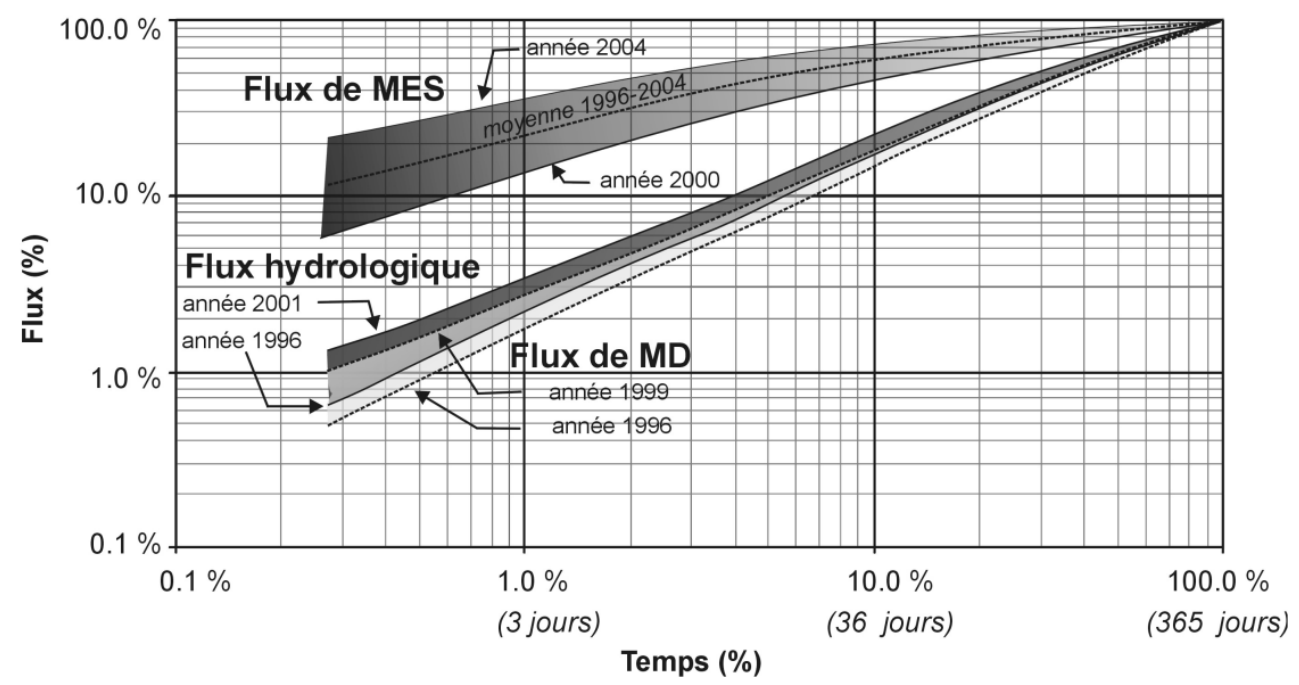

Figure 4. Courbes enveloppes de durée annuelle: pourcentage du temps nécessaire pour évacuer une quantité du flux hydrologique, des flux annuels de matières dissoutes et particulaires. Pour la période 1996-2004, toutes les courbes sont «empilées 》 les unes sur les autres, et inscrites dans trois enveloppes graphiques définies pour chacun des éléments. Les courbes de durée annuelle des flux de MES, des années 1995 et 2005, sont singulières et ne sont pas représentées sur le graphique.

Figure 4. Yearly ranges: the time in percentage points of a year (with the equivalent number of days) needed to evacuate a given volume of water, annual dissolved matter and particulate flows. For the period from 1996 to 2004, lines have been "stacked", and inserted within a band showing the range for each of the three parameters. Times for annual SM flows were abnormal between 1995 and 2005, and are not shown on the graph.

\section{Variations saisonnières et interannuelles}

En n'intégrant pas les années 1995 et 2005, les flux annuels de MES s'échelonnent de 0.89 à 3.16 $\mathrm{Mt} \mathrm{an}^{-1}$ (tableau 5), avec des flux mensuels compris entre $0.12 \mathrm{Mt}$ (janvier 2002) à $1.78 \mathrm{Mt} \mathrm{mois}^{-1}$ (en mai 1999). Les flux mensuels varient donc dans un rapport de 1 à 15, contre une variation de 3.5 pour 
les flux annuels. Ces gammes de variations traduisent la grande variabilité interannuelle de ces flux et soulignent l'importance des fluctuations saisonnières, bien plus grandes que les fluctuations interannuelles.

En 1995 et 2005, les flux de sédiments mesurés (de 5.7 et $8.1 \mathrm{Mt} \mathrm{an}^{-1}$ ) sont pour l'essentiel consécutifs à des perturbations anthropiques, engendrant des transits annuels anormaux. Afin de cerner les flux annuels de MES que l'on aurait pu observer en 1995 et 2005, sans ces perturbations, et aussi d'apprécier ces transits sur une période plus large, plusieurs modèles ont été testés, mais feront l'objet d'études complémentaires. En effet, les simples relations du type $M E S=a Q^{b}$, systématiquement utilisées dans les études (Eisma, 1993 ; Meddi, 1999 ; Meybeck, 2001 ; Pont et al., 2002 ; Antonelli, 2002), demeurent sur les données journalières de l'Isère médiocrement établies, sauf exceptions. En revanche, à l'échelle annuelle, il est possible de cerner assez convenablement le transit de MES ( $\mathrm{Fa}_{\mathrm{MES}}$, en $\mathrm{Mt} \mathrm{an}^{-1}$ ) à partir de l'écoulement annuel $\left(\mathrm{Qa}\right.$, en $\left.\mathrm{m}^{3} \mathrm{~s}^{-1}\right)$, avec la relation (3) suivante (Mohamed Bourouba, 2003) :

$$
\mathrm{Fa}_{\mathrm{MES}}=0.0276 \mathrm{Qa}-3.0531 \mathrm{R}=0.88 \text { (équation 3) }
$$

Tableau 5. Concentration et flux de MES annuels mesurés et calculés Table 5. Concentration and flow of annual calculated and measured SM

\begin{tabular}{|c|c|c|c|c|}
\hline Année / Période & $\begin{array}{c}\text { Module } \\
(\mathrm{m} 3 / \mathrm{s})\end{array}$ & $\begin{array}{c}\text { Concentration } \\
\text { moyenne } \\
(\mathrm{mg} / \mathrm{L})\end{array}$ & $\begin{array}{c}\text { Flux mesuré } \\
\text { (tonnes/an) }\end{array}$ & $\begin{array}{c}\text { Flux calculé } \\
\text { Qs=f(Q) } \\
\text { (tonnes/an) }\end{array}$ \\
\hline 1995 & 235 & 489 & 5741198 & 3423653 \\
\hline 1996 & 145 & 176 & 1122881 & 958741 \\
\hline 1997 & 148 & 157 & 911221 & 1034396 \\
\hline 1998 & 158 & 164 & 996045 & 1315700 \\
\hline 1999 & 213 & 293 & 3162337 & 2832097 \\
\hline 2000 & 168 & 177 & 1163197 & 1586589 \\
\hline 2001 & 218 & 284 & 3017795 & 2973072 \\
\hline 2002 & 163 & 145 & 894946 & 1455102 \\
\hline 2003 & 147 & 176 & 965475 & 998871 \\
\hline 2004 & 140 & 240 & 1657047 & 811853 \\
\hline 2005 & 129 & 1678 & 8085953 & 507641 \\
\hline Moy. 1995-2005 & 170 & & & 1627065 \\
\hline Moy. 1996-2004 & 167 & 201 & 1543438 & 1551825 \\
\hline
\end{tabular}

A partir de cette estimation des flux de MES, il semble probable que les aménagements du cours d'eau ont produit un excès de sédiments par rapport à ce que l'on pouvait attendre, de plus de $2.3 \mathrm{Mt}$, en 1995, et de plus 7.5 Mt, en 2005 (tableau 5). Sur la période 1995-2005, avec une estimation basée sur l'écoulement annuel pour ces deux années, l'exportation moyenne est de $1.62 \mathrm{Mt} \mathrm{an}^{-1}$ (tableau 5). Néanmoins sur cette période, l'hydraulicité moyenne de l'Isère, avec $170 \mathrm{~m}^{3} \mathrm{~s}^{-1}$, est alors légèrement inférieure à celle définie sur 46 années (moyenne de 1960 à $2005: 183 \mathrm{~m}^{3} \mathrm{~s}^{-1}$ ), plus représentative de l'écoulement moyen de l'Isère. Pour ce module, le transit annuel estimé à partir de l'équation 3 , serait de $2.0 \mathrm{Mt} \mathrm{an}^{-1}$, le taux d'érosion moyen de $349 \mathrm{t} \mathrm{km}^{-2} \mathrm{an}^{-1}$ (tableau 6).

\section{Bilan sédimentaire et synthèse}

Ces différentes quantifications permettent de dresser un bilan sédimentaire pour l'Isère (tableau 6). La grande stabilité des sections de l'Isère à Grenoble (Dumas 2004b), les études du transit sédimentaire à hauteur de sa confluence avec le Drac (Salvador, 1991), les travaux sur les profils de l'Isère dans la vallée du Grésivaudan (Vautier, 2000, Allain Jegou, 2002) montrent que la charge de fond n'est plus exportée à la fin du XX $X^{e}$ siècle. La charge de fond étant négligeable (Vautier, 2000, Allain Jegou, 2002), voire nulle, cette synthèse cerne bien l'ensemble du transit sédimentaire de l'Isère. La répartition des flux annuels de MES et de MDT est sensiblement équivalente, respectivement de 2.0 $\mathrm{Mt} \mathrm{an}^{-1}$ et de $1.73 \mathrm{Mt} \mathrm{an}^{-1}$ (tableau 6). Le flux annuel de MES devient véritablement prépondérant sur le transit de MDT lors de crues importantes. 
Les dégradations spécifiques annuelles associées aux flux de MES sont inscrites dans la fourchette de 89 à $599 \mathrm{t} \mathrm{km}^{-2} \mathrm{an}^{-1}$, avec une dégradation spécifique moyenne de $349 \mathrm{t} \mathrm{km}^{-2} \mathrm{an}^{-1}$. Ce taux d'érosion moyen calculé pour l'Isère masque une grande hétérogénéité spatiale des apports sédimentaires. L'Arc, avec notamment son affluent l'Arvan, est de loin le plus gros pourvoyeur de I'Isère en MES (Marnezy, 1999). La dégradation spécifique moyenne de l'Arvan se situe au-dessus de $2500 \mathrm{t} \mathrm{km}^{-2} \mathrm{an}^{-1}$, pour un bassin torrentiel d'environ $200 \mathrm{~km}^{2}$, elle passe à la confluence de l'Arc avec I'Isère, avec un bassin de près de $2000 \mathrm{~km}^{2}$, à $766 \mathrm{t} \mathrm{km}^{-2} \mathrm{an}^{-1}$, avec un flux annuel moyen de 1.5 $M t \mathrm{an}^{-1}$ d'exportation de MES (Marnezy, 1999). Cette différence n'a rien d'exceptionnel sur un bassin de grande dimension (Meybeck et al., 2003), inscrit de surcroît sur une zone alpine à forte complexité lithologique, favorisant inévitablement une diversité géographique des apports sédimentaires. Sur le bassin de l'Isère, la zone la plus favorable à la recharge sédimentaire est justement la vallée de la Maurienne (vallée de l'Arc) où dominent des zones peu végétalisées et des formations géologiques sensibles à l'érosion ; Lias schisteux sur la rive gauche de l'Arc (bassin de l'Arvan en particulier) et schistes lustrés en amont de Modane.

Le taux d'érosion spécifique moyen de l'Isère s'intègre néanmoins parfaitement dans la zonation de 250 à $500 \mathrm{t} \mathrm{km}^{-2} \mathrm{an}^{-1}$ proposée pour la bordure est-pyrénéenne (Walling et Webb, 1996 ; Serrat, 1999 ; Serrat et al., 2001), mais dépasse de beaucoup la moyenne européenne comprise entre 30 et $80 \mathrm{t} \mathrm{km}^{-2} \mathrm{an}^{-1}$ (Collins, 1986 ; Ludwig et Prosbt, 1998). II rejoint les valeurs de certains grands cours d'eau alpins avec des bassins d'une superficie relativement comparable (Schlunegger et Hinderer, 2001 ; Schlunegger et Hinderer, 2003 ; Meybeck et al., 2003) ; par exemple en Suisse, celui de l'Arve à Genève $\left(299 \mathrm{t} \mathrm{km}^{-2} \mathrm{an}^{-1}\right.$ avec un bassin de $\left.2079 \mathrm{~km}^{2}\right)$, du Rhône alpin à la Porte de Scex $(320 \mathrm{t} \mathrm{km}$ ${ }^{2} \mathrm{an}^{-1}$ avec un bassin de $5220 \mathrm{~km}^{2}$ ), ou encore du Rhin alpin à Bad Ragaz (323 $\mathrm{t} \mathrm{km}^{-2} \mathrm{an}^{-1}$ avec un bassin de $4455 \mathrm{~km}^{2}$ ). La dégradation spécifique moyenne de l'Isère rejoint également, voire même dépasse certaines années, celle de la Durance, évaluée à $360 \mathrm{t} \mathrm{km}^{-2} \mathrm{an}^{-1}$ (Alary, 1998), cours d'eau de l'arc alpin, mais située dans un contexte beaucoup plus méditerranéen.

Au niveau de l'Arc alpin, c'est justement dans les Alpes du Sud, et tout particulièrement dans les marnes noires, que les taux d'érosion observés sur des parcelles ou de petits bassins versants sont les plus forts, et généralement compris entre 12500 et $20000 \mathrm{t} \mathrm{km}^{-2} \mathrm{an}^{-1}$ (Descroix et Olivry, 2002). Ils peuvent même dépasser localement $60000 \mathrm{t} \mathrm{km}^{-2} \mathrm{an}^{-1}$ (Lhénaff et al., 1993). Cette forte variabilité spatiale de l'érosion spécifique se retrouve également dans les Alpes du Nord (Vivian, 1981 ; Descroix et Gautier, 2002 ; Descroix et Mathys, 2003), où le remplissage sédimentaire de barrages indique des taux d'érosion inégaux; $670 \mathrm{t} \mathrm{km}^{-2} \mathrm{an}^{-1}$ au barrage du Sautet (bassin de $1000 \mathrm{~km}^{2}$ ), $490 \mathrm{t} \mathrm{km}^{-2} \mathrm{an}^{-1} \mathrm{au}$ barrage du Verney (bassin de $120 \mathrm{~km}^{2}$ ), $220 \mathrm{t} \mathrm{km}^{-2} \mathrm{an}^{-1}$ au barrage du Chambon (bassin de $220 \mathrm{~km}^{2}$ ), et atteint même $90 \mathrm{t} \mathrm{km}^{-2} \mathrm{an}^{-1}$ au barrage d'Aussois (bassin de $150 \mathrm{~km}^{2}$ ). Cette variabilité spatiale de l'érosion se retrouve également à l'échelle du cours d'eau lui-même, elle est décrite, par exemple, au NW de l'Italie, sur un affluent alpin du Pô, la Dora Baltea. Les transferts sédimentaires de MES sont plus marqués dans la partie supérieure de son bassin et diminuent vers l'aval, avec des valeurs d'érosion spécifique de $553 \mathrm{t} \mathrm{km}^{-2} \mathrm{an}^{-1}$ sur un bassin de $543 \mathrm{~km}^{2}$, de $265 \mathrm{t} \mathrm{km}^{-2} \mathrm{an}^{-1}$, plus en aval, sur un bassin de $1303 \mathrm{~km}^{2}$, et de $181 \mathrm{t} \mathrm{km}^{-2} \mathrm{an}^{-1}$ sur un bassin de $3264 \mathrm{~km}^{2}$ (Vezzoli, 2004).

Sur le bassin de l'Isère, les tranches d'érosion moyennes correspondantes, basées sur une densité de $2.5^{1}$, montrent un taux de dénudation spécifique moyen du bassin versant de $0.26 \mathrm{~mm} \mathrm{an}^{-1}$ (tableau 6). L'extension du bassin versant engendre une diminution, d'un rapport proche de 2 , de la tranche érodée estimée en amont, sur le bassin de l'Arc, de presque $2000 \mathrm{~km}^{2}$, avec une érosion globale évaluée à $0.62 \mathrm{~mm} \mathrm{an}^{-1}$ (Marnezy, 1999). Sur le bassin de l'Isère, l'érosion fluviatile moyenne est de $0.14 \mathrm{~mm} \mathrm{an}^{-1}$, et l'ablation par dissolution égale à $0.12 \mathrm{~mm} \mathrm{an}^{-1}$ (tableau 6). Ces résultats sont à rapprocher des 0.1 à $0.17 \mathrm{~mm} \mathrm{an}^{-1}$ donnés pour la dissolution des calcaires dans les Alpes du Nord (Chardon, 1996), et restent bien inférieurs à ceux obtenus directement sur les gypses, où les taux de dissolution sont égaux ou supérieurs à $1 \mathrm{~mm} \mathrm{an}^{-1}$ (Rovera, 1990). Ils rejoignent également l'estimation de l'érosion fluviatile de 0.25 à $0.45 \mathrm{~mm} \mathrm{an}^{-1}$ sur les bassins de la Romanche et du Drac, proposée à partir de mesures de dépôts dans les barrages (Sikirdji et al., 1982). Mais largement au-dessous des vitesses d'érosion de $10 \mathrm{~mm}$ an $^{-1}$ estimées dans des bassins inscrits dans des marnes et situés dans les Alpes du Sud (Descroix, 1994).

\footnotetext{
${ }^{1}$ Les densités utilisées différent cependant légèrement selon les études, par exemple ; $\mathrm{d}=2.4$ pour la MD, Chardon, 1996 et Marnezy, 1999 ; d=2.5 pour la MES, Serrat, 1999 ; d=2 pour la MES et la charge de fond, Vautier 2000.
} 
Tableau 6. Bilan sédimentaire annuel

Table 6. Annual sedimentation rates

\begin{tabular}{|c|c|c|c|c|c|c|c|c|c|c|}
\hline Année & $\begin{array}{c}\text { Module } \\
(\mathrm{m} 3 / \mathrm{s})\end{array}$ & $\begin{array}{c}\text { Flux de } \\
\text { MES } \\
\text { mesuré ou } \\
\text { calculé }\left(^{\star}\right) \\
\text { (Mt/an) }\end{array}$ & $\begin{array}{c}\text { Flux de MD } \\
\text { mesuré ou } \\
\text { calculé }\left(^{\star}\right) \\
(\mathrm{Mt} / \mathrm{an})\end{array}$ & $\begin{array}{c}\text { Flux } \\
\text { total } \\
\text { (Mt/an) }\end{array}$ & $\begin{array}{c}\text { Flux } \\
\text { spécifique } \\
\text { de MES } \\
\text { (t/km²/an) }\end{array}$ & $\begin{array}{c}\text { Flux } \\
\text { spécifique } \\
\text { de MD } \\
\text { (t/km²/an) }\end{array}$ & $\begin{array}{c}\text { Flux } \\
\text { spécifique } \\
\text { total } \\
\text { (t/ } / \mathbf{k m}^{2} / \text { an) }\end{array}$ & $\begin{array}{c}\text { Iaux } \\
\text { d'érosion } \\
\text { spécifique } \\
\text { de MES } \\
\text { (d=2.5) } \\
\text { (mm/an) }\end{array}$ & $\begin{array}{c}\text { I aux } \\
\text { d'érosion } \\
\text { spécifique } \\
\text { de MD } \\
(\mathrm{d}=2.5) \\
\text { (mm/an) }\end{array}$ & $\begin{array}{c}\text { Taux } \\
\text { d'érosion } \\
\text { spécifique } \\
\text { total } \\
\text { (mm/an) }\end{array}$ \\
\hline 1995 & 235 & ${ }^{*} 3.42$ & ${ }^{*} 2.04$ & 5.46 & 599 & 357 & 955 & 0.24 & 0.14 & 0.38 \\
\hline 1996 & 145 & 1.12 & * 1.48 & 2.60 & 196 & 259 & 455 & 0.08 & 0.10 & 0.18 \\
\hline 1997 & 148 & 0.91 & * 1.49 & 2.40 & 159 & 260 & 419 & 0.06 & 0.10 & 0.17 \\
\hline 1998 & 158 & 1.00 & * 1.56 & 2.56 & 174 & 273 & 448 & 0.07 & 0.11 & 0.18 \\
\hline 1999 & 213 & 3.16 & * 1.91 & 5.07 & 553 & 334 & 887 & 0.22 & 0.13 & 0.35 \\
\hline 2000 & 168 & 1.16 & 1.67 & 2.84 & 203 & 292 & 496 & 0.08 & 0.12 & 0.20 \\
\hline 2001 & 218 & 3.02 & 1.93 & 4.95 & 528 & 338 & 866 & 0.21 & 0.14 & 0.35 \\
\hline 2002 & 163 & 0.89 & 1.64 & 2.54 & 156 & 287 & 443 & 0.06 & 0.11 & 0.18 \\
\hline 2003 & 147 & 0.97 & 1.50 & 2.47 & 169 & 263 & 431 & 0.07 & 0.11 & 0.17 \\
\hline 2004 & 140 & 1.66 & 1.37 & 3.03 & 290 & 240 & 530 & 0.12 & 0.10 & 0.21 \\
\hline 2005 & 129 & ${ }^{*} 0.51$ & 1.34 & 1.85 & 89 & 235 & 324 & 0.04 & 0.09 & 0.13 \\
\hline oyenne & - 170 & - 1.62 & . & 3.25 & 283 & -285. & 568 & 0.11 & -0.11 & 0.23 \\
\hline pour un débit & 183 & ${ }^{*} 2.00$ & ${ }^{*} 1.73$ & 3.73 & 349 & 303 & 652 & 0.14 & 0.12 & 0.26 \\
\hline
\end{tabular}

\section{Evolution des flux sédimentaires depuis le début du XIXe siècle}

L'estimation de la dynamique sédimentaire actuelle, évaluée à $2 \mathrm{Mt} \mathrm{an}^{-1}$, permet également d'apporter une nouvelle lecture de la tendance générale du transit sédimentaire, depuis le $\mathrm{XIX}^{\mathrm{e}}$. En effet, Allain Jegou (2002), en complétant les travaux de Vautier (2000), et comme ce dernier, note une décroissance très importante des flux de matière solide, entre le début du $X I X^{e}$ et la fin du $X X^{e}$ siècle. Au début du XIX ${ }^{e}$, le débit solide, "incluant charriage et suspension ", avoisinait $0.4 \mathrm{Mt} \mathrm{an}^{-1}$ (Allain Jegou, 2002). Actuellement, mais en supposant une exportation de sédiments très faible, voire nulle, à l'aval de Grenoble, les auteurs estiment les flux annuels moyens à environ $0.02 \mathrm{Mt} \mathrm{an}^{-1}$, pour la période 1984-1990, et à $0.16 \mathrm{Mt} \mathrm{an}{ }^{-1}$ pour la période 1990-2000, dont $75 \%$ seraient liés au transit en suspension (ces valeurs se basent sur les valeurs volumétriques estimées par Vautier, 2000 et Allain Jegou, 2002).

Outre le fait que ces estimations se basent sur l'hypothèse d'un débit solide négligeable à l'aval de Grenoble qui pose problème à nos yeux, les auteurs ne distinguent pas charge de fond et transit de MES, seule la charge de fond semble bien ne plus être exportée (Salvador, 1991 ; Peiry et al., 1994). Les nombreux seuils édifiés dans le lits des cours d'eau bloqueraient les transports solides, à tel point qu'il n'y aurait plus de matériaux grossiers parvenant à la confluence Drac-Isère (Peiry et al., 1994). Le transit sédimentaire en suspension de l'Isère, avec un flux annuel moyen estimé pour ces dernières décennies à $2.0 \mathrm{Mt} \mathrm{an}^{-1}$, en revanche, reste très important. II s'avère donc difficile de négliger l'exportation des matériaux en suspension au niveau de Grenoble au risque sinon de conclure comme ces auteurs à une diminution du transit sédimentaire depuis le $\mathrm{XIX}^{\mathrm{e}}$ siècle ; conclusion ensuite reprise dans certains travaux (Descroix et al., 2002). Pour les deux périodes précédentes (1984-1990 et 1990-2000), dans le cadre de cette étude, les flux moyens de MES sont estimés respectivement à $2.13 \mathrm{Mt} \mathrm{an}^{-1}$, et à $1.89 \mathrm{Mt} \mathrm{an}^{-1}$ (moyennes calculées avec l'équation 3). Le dépôt sédimentaire actuel dans le lit, de $0.02 \mathrm{Mt} \mathrm{an}^{-1}$ pour la période 1984-1990 (Vautier, 2000), et de $0.16 \mathrm{Mt} \mathrm{an}^{-1}$ pour la période 1990-2000 (Allain Jegou, 2002), ne modifie donc que peu le débit solide moyen annuel, et laisse supposer, à l'inverse des conclusions apportées par ces auteurs (Vautier, 2000, Allain Jegou, 2002), que le transit sédimentaire de MES aurait eu plutôt tendance à s'accroître, dans un rapport de 1 à 5 depuis le $X \mathrm{XX}^{e}$ siècle (charge de fond et matière en suspension alors évaluées à $0.4 \mathrm{Mt} \mathrm{an}^{-1}$ ). Dans l'ensemble des Alpes françaises, entre 1860 et 1928 , suite aux analyses du " grand ingénieur " Surell, les importants reboisements entrepris par l'Administration des Eaux et Forêts (Pardé, 1964), et ensuite depuis les années 1950, la dynamique de reboisement naturel liée à la déprise rurale généralisée, pourraient bien sûr avoir diminué ces flux. Les prélèvements de galets jusqu'en 1970 ont aussi contribué à modifier notablement le transit des matériaux (Vautier, 2000). De même, les grands aménagements du bassin de l'Isère, dans les années 70 (Bissorte, Chevril, Girotte, Roselend, Mont-Cenis, dérivation du Cheylas...) ont créé une nouvelle donne sur les débits solides, en écrêtant les débits de pointe des crues fréquentes, notamment lors de la fusion nivale. Certes, il est bien connu que ces aménagements hydrauliques ont fortement modifié les écoulements liquides et solides (Ackers et Thompson, 1987 ; Reid et Frostick, 1994 ; Milliman, 
1997 ; Marnezy, 1999). Sur le bassin du Rhône, qui compte actuellement 78 barrages, si le transit de la charge de fond reste toujours très réduit depuis leur installation progressive (Donzère est le premier en 1952), en revanche, les perturbations sur le transit de la matière en suspension semblent avoir été temporaires, et ont progressivement diminué depuis les années 50 (Pont et al., 2002). Par ailleurs, les grandes crues, peu touchées par les aménagements hydroélectriques, restent de nos jours probablement toujours aussi efficaces pour le transit de sédiments, et si les écoulements ont perdu dans la zone amont une partie de leur charge en suspension par décantation (barrages-réservoirs et retenues au fil de l'eau), en revanche les rythmes diurnes d'écoulement plus marqués aujourd'hui (la dérivation du Cheylas, par exemple, engendre à Grenoble des variations quotidiennes de plus $100 \mathrm{~m}^{3}$ $\mathrm{s}^{-1}$ ), la diminution de la charge de fond en amont (Marnezy, 1999), renforcent probablement l'efficacité globale d'exportation de MES par les écoulements, et expliqueraient l'augmentation du transit depuis le début du XIX ${ }^{\mathrm{e}}$ siècle.

\section{CONCLUSION}

Les résultats présentés sont les premiers établis sur un important cours d'eau alpin à partir de mesures systématiques. Dans les Alpes, il n'existe encore que peu de données continues et relevées sur une décennie. A l'échelle de ce bassin, et aux pas de temps annuel et mensuel, les flux de sédiments sont fortement dépendants des écoulements. Le taux d'érosion spécifique moyen de I'Isère, évalué à $652 \mathrm{t} \mathrm{km}^{-2} \mathrm{an}^{-1}$, dépasse celui des Pyrénées orientales, 250-500 t km${ }^{-2} \mathrm{an}^{-1}$ (Walling et Webb, 1996).

L'érosion fluviatile moyenne avec $2.0 \mathrm{Mt} \mathrm{an}^{-1}$ est légèrement plus forte que celle issue des processus chimiques dont la valeur moyenne est estimée à $1.73 \mathrm{Mt} \mathrm{an}^{-1}$. L'exportation de matière dissoute est cependant considérable et traduit l'importance des processus de dissolution au sein du bassin. Dans la vallée du Grésivaudan, l'exhaussement moyen du lit de l'Isère correspondrait à une sédimentation moyenne, calculée sur la période 1990-2000, de $0.16 \mathrm{Mt} \mathrm{an}^{-1}$, et dont $75 \%$ seraient liées au transit en suspension (ces valeurs se basent sur les valeurs volumétriques estimées par Allain Jegou, 2002). La vitesse moyenne d'érosion mécanique réelle, tout en restant dans la même gamme de valeurs, serait donc légèrement plus forte (exportation totale de $2.16 \mathrm{Mt} \mathrm{an}^{-1}$ ).

\section{Remerciements}

L'auteur remercie J.-L. Peiry (professeur à l'université de Clermont-Ferrand) pour lui avoir transmis une partie de l'équipement pour la mesure de la MES et de la MDT à la station Grenoble-Campus, $P$. Bois (professeur à l'INPG) et l'INPG pour les facilités d'accès à la station hydrologique du Campus. L'auteur remercie également M. Mietton (professeur à l'université de Lyon), M. Chardon (professeur émérite à l'université de Grenoble), J.-P. Charre (maître de conférences à l'université de Grenoble) et les relecteurs anonymes pour leurs suggestions et remarques, qui ont contribué à améliorer le manuscrit.

\section{RÉFÉRENCES}

Ackers, P. \& Thompson, G. (1987) : Reservoir sedimentation and influence of flushing. In Sediment Transport in Gravel-bed Rivers (C.R. Thorne, J.C. Bathurst, R.D. Hey eds), John Wiley and Sons, Chichester, pp. 845-861.

Alary, C. (1998) : Mécanismes et bilans d'érosion dans un bassin versant méditerranéen aménagé : le cas de la Durance (S-E France). - Thèse, Univ. d'Aix-Marseille, $276 \mathrm{p}$.

Allain Jegou, C. (2002) : Relations végétation, écoulement, transport solide dans le lit des rivières ; étude de l'Isère dans le Grésivaudan. - Thèse, Inst. Nat. Polytech. de Grenoble, spécialité Mécanique des milieux géophysiques et environnement, $198 \mathrm{p}$.

Antonelli, C. (2002) : Flux sédimentaires et morphogenèse récente dans le chenal du Rhône aval. Thèse, université d'Aix-Marseille I, $272 \mathrm{p}$.

Batalla A., Sala M. \& Werritty A. (1995) : Sediment budget focused in solid transport in a subhumid Mediterranean drainage basin. Zeitschhrift für Geomorphologie, 39, 2, 249-264.

Chardon, M. (1996) : La mesure de l'érosion dans le gypse/anhydrite des Alpes françaises du Nord. Méthodes et état des connaissances. - Revue de Géographie Alpine, 2, 84, 45-56.

Collins, M.B. (1986) : Processes and controls involved in the transfer of fluviatile sediments to the deep ocean. J. Geol. Soc., London 143, 915-920. 
Descroix, L. \& Gautier, E. (2002) : Water erosion in the southern French alps: climatic and human mechanisms. Catena 50, 53-85.

Descroix, L. \& Mathys, N. (2003) : Processes, spatio-temporal factors and measurements of current erosion in French Southern Alps : a review. Earth Surface Processes and Landforms 28, 993-1011.

Descroix, L. \& Olivry, J.-C., (2002) : Spatial and temporal factors of erosion by water of black marls in the badlands of the French southern Alps. Hydrological Sciences-J.-des Sciences Hydrologiques 47, $2,227-242$.

Descroix, L. (1994) : L'érosion actuelle dans la partie occidentale des Alpes du Sud. - Thèse, Univ. Lyon II, $353 \mathrm{p}$

Dumas, D. (2004a) : Optimisation de la quantification des flux de matière en suspension d'un cours d'eau alpin : I'Isère à Grenoble (France). - Comptes Rendus Geosciences Volume 336, Issue 13 11491159.

Dumas, D. (2004b) : Les deux crues mémorables de l'Isère à Grenoble (1651 et 1859) : Analyse des estimations de M. Pardé. - Revue de Géographie Alpine, Journal of Alpine Research, n³, 27-38.

Eisma, D. (1993) : Suspended matter in the aquatic environment. - Springer-Verlag, Berlin, $315 \mathrm{p}$.

Lhénaff, R., Coulmeau, P., Lecompte, M. \& Marre, A. (1993) : Erosion and transport processes on badlands slopes in the Baronnies mountains (French Southern Alps). Geografia Fisica e Dinâmica Quaternaria 16, 65-73.

Ludwig, W. \& Probst, J.-L. (1998) : River sediment discharge to the oceans:present-day controls and global budgets, Am. J. Sci 298, 265-295.

Marnezy, A. (1999) : L'Arc et sa vallée. Anthropisation et géodynamique d'une rivière alpine dans son bassin versant. - Thèse d'Etat, Univ. Joseph Fourier, Grenoble I, $682 \mathrm{p}$.

Martin, C. (1987): Les mesures de l'érosion chimique dans les bassins-versants de roches cristallines: comparaison des résultats obtenus par différentes méthodes d'investigation dans le massif des Maures (Var, France). - Zeitschhrift für Geomorphologie, 31, 1, 73-84.

Meddi, M. (1999) : Etude du transport solide dans le bassin versant de l'oued Ebda (Algérie). Zeitschhrift für Geomorphologie, 43,2, 167-183.

Meybeck, M. (2001) :Transport et qualité des sédiments fluviaux : de la variabilité spatio-temporelle à la gestion. - La Houille Blanche, 6-7, 34-43.

Meybeck, M., Laroche, L., Dürr, H.H. \& Syvitski, J.P.M. (2003) : Global variability of daily total

suspended solids and their fluxes in rivers. - Global and Planetary Change, 39, 65-93.

Milliman, J.D. (1997) : Blessed dams or damned dams? Nature, 386, 325-327.

Mohamed Bourouba, R. (2003) : Etude de la teneur de sédiments en suspension de deux oueds méditerranéens intramontagneux du Tell oriental (Algérie). - Zeitschhrift für Geomorphologie, 47,1, 5181.

Pardé, M. (1964) : Fleuves et rivières. Coll. Armand Colin, $n^{\circ} 155$, section de Géographie, 4ème édition, $224 \mathrm{p}$.

Peiry, J.-L. (1997) : Recherches en géomorphologie fluviale dans les hydrosystèmes fluviaux des Alpes du Nord. - IGA Habilitation, Université Joseph-Fourier, Grenoble-1, 308 p.

Peiry, J.-L., Salavador, P.-G. \& Nougier, F. (1994) : L'incision des rivières dans les Alpes du nord : état de la question. Rev. de Géographie de Lyon, vol. 69, 47-56.

Pont, D., Simonnet, J.P. \& Walter, A.V. (2002) : Medium-term changes in suspended sediment delivery to the ocean consequences of catchment heterogeneity and river management (Rhône River, France). Estuar Coast Shelf S. 54, 1-18.

Reid, I. \& Frostick, L.E. (1994) : Fluvial sediment transport and deposition. In Sediment Transport and Deposition Processes (K. Pye, ed.), Blackwell Scientific Publications, Oxford, pp. 89-156.

Rodier, J. (1996) : L'analyse de l'eau : eaux naturelles, eaux résiduaires, eau de mer. - Paris Dunod, 8è éd. $1434 \mathrm{p}$.

Rovera, G. (1990): Géomorphologie dynamique et aménagement des versants en moyenne Tarentaise. Thèse, Univ. J. Fourier, Institut de Géographie Alpine, $465 \mathrm{p}$.

Salvador, P.G. (1991) : Le thème de la métamorphose fluviale dans les plaines alluviales du Rhône et de l'Isère. - Thèse de Goégraphie, Univ. Lyon III, $498 \mathrm{p}$.

Schlunegger, F. \& Hinderer, M. (2001) : Crustal uplift in the Alps: why the drainage pattern matters. Terra Nova 13, 425-432.

Schlunegger, F. \& Hinderer, M. (2003) : Pleistocene/Holocene climate change, re-establishment of fluvial drainage network and increase in relief in the Swiss Alps. Terra Nova 15, 88-95.

Serrat, P. (1999): Dynamique sédimentaire actuelle d'un système fluvial méditerranéen: I'Agly (France). - C. R. Acad. Sci. Paris, Ser. Ila 329, 3, 189-196. 
Serrat, P., Ludwig, W., Navarro, B. \& Blazi, J.-L. (2001) : Variabilité spatio-temporelle des flux de matière en suspension d'un fleuve côtier méditerranéen : la Têt (France). - C. R. Acad. Sci. Paris, série lla 333, 389-397.

Sikirdji L., Fabre, D. \& Giraud, A. (1982) : L'envasement de la retenue du Chambon (Alpes françaises) après un demi-siècle d'exploitation. - IRIGM, Grenoble, $10 \mathrm{p}$.

Vautier, F. (2000) : Dynamique géomorphologique et végétalisation des cours d'eau endigués : l'exemple de l'Isère dans le Grésivaudan. - Thèse, Institut de Géographie alpine, Grenoble, 408 p.

Vezzoli, G. (2004) : Erosion in the Western Alps (Dora Baltea Basin) : 2. Quantifying sediment yield. Sedimentay Geology 171, 247-259.

Vivian, H. (1969) : Les crues de l'Isère à Grenoble et l'aménagement actuel des digues. Revue de Géographie Alpine, tome LVIII, 1, 53-84.

Vivian, H. (1981) : Erosion et transports solides dans le bassin du Drac au Sautet. Doc. du BRGM, séminaire national Propriano (Corse) : la gestion régionale des sédiments, 249-357.

Walling, D.D., Webb, D.W. \& Woodward, J.C. (1992) : Some sampling considerations in the design of effective stategies for monitoring sediment-associated transport. - IAHS Public., 210, 279-288.

Walling, D.D., Webb, D.W. (1996) : Erosion and sediment Yield: Global and Regional Perspectives. IAHS., 236, 3-19. 